\title{
A Quality of Experience Handover System for Heterogeneous Multimedia Wireless Networks
}

\author{
Carlos Quadros, \\ Eduardo Cerqueira \\ Federal University of Para \\ Belém, Brazil \\ \{jean, cerqueira\}@ufpa.br
}

\author{
Augusto Neto \\ Federal University of Ceara \\ Fortaleza, Brazil \\ augusto@deti.ufc.br
}

\author{
Antonio Pescapé \\ University of Napoli Federico \\ II \\ Naples, Italy \\ pescape@unina.it
}

\author{
Andre Riker, Roger Immich, \\ Marilia Curado \\ University of Coimbra \\ Coimbra, Portugal \\ \{riker, \\ immich,marilia $\} @$ dei.uc.pt
}

\begin{abstract}
The convergence of emerging real-time multimedia services, the increasing coverage of wireless networks and the ever-growing popularity of mobile devices, are leading to an era of user-centric multimedia wireless services. In this scenario, heterogeneous communications will co-exist and ensure that the end-user is always best connected. However, the Quality of Experience (QoE) support for emerging video applications in multi-operator environments remains a significant challenge and is crucial for the success of wireless multimedia systems. This paper presents a Quality of Experience Handover Architecture for Converged Heterogeneous Wireless Networks, called QoEHand. QoEHand allows users of multimedia content to be always best connected in IEEE 802.11e and IEEE 802.16e environments. Simulation results show the impact and benefit of the proposed solution in multi-access and multi-operator wireless scenarios by using objective and subjective QoE metrics.
\end{abstract}

Keywords: Video Streaming; QoE; Wireless networks.

\section{INTRODUCTION}

The development of new real-time multimedia services, the increasing coverage of heterogeneous wireless networks and the ever-growing popularity of mobile devices, are changing the life-style of users and creating a user-centric multimedia wireless era. In addition, the integration of heterogeneous networks, such as IEEE 802.11 and IEEE 802.16, in multiaccess and multi-operator systems, is bringing about revolutionary changes in the Internet by providing new opportunities, introducing better communication channels and raising possibility of providing better Quality of Experience (QoE) assurance for users of wireless services. Emerging multi-access/operator wireless environments will allow clients to be connected to the best QoE-aware wireless access network, where seamless mobility will be combined with respect for the user's preferences. In this scenario, the creation of novel architectures is required to allow vertical and horizontal QoE-aware handovers in heterogeneous networks and an estimation of the impact of QoS/QoE is needed [12]. In order to assure connectivity and mobility in heterogeneous systems, the IEEE 802.21/Media Independent Handover Services (MIH) standard was proposed [1], but MIH alone does not provide QoE support for multimedia applications. Due to the heterogeneity of future wireless systems (networks different service classes and applications, as well as links with different capacities), novel QoE architectures (including $\mathrm{MIH}$ ) with quality estimator, mapping and adaptation mechanisms, are needed to provide QoE support for real-time 2D/3D video streaming in multi-operator and multi-access environments [23]. One requirement is to improve the handover decision process by assessing the QoE of users consuming video flows. Another issue is that each access network must support different QoS models (e.g., IEEE 802.11e or IEEE 801.16), and offer the same wireless service classes with different definitions or even service classes with different compositions [4]. Therefore, a QoE mapping must be used to map application requirements and user's perception into available wireless service classes based on information about the available classes (within or between networks) and the predicted QoE given by the quality estimator. Moreover, in periods of congestion in a selected service class, an adaptation scheme must adjust the quality level of video flows, by selecting a different class to map multimedia packets or by dropping packets in overloaded queues in accordance with the impact they have on the user's experience.

This paper extends MIH with a Quality of Experience Handover Architecture for Converged Heterogeneous Wireless Networks (QoEHand) to allow users of multimedia content to be always best connected in IEEE 802.11e and IEEE 802.16e environments. QoEHand coordinates video quality estimator, mapping and adaptation components in order to provide QoEaware seamless handovers in multi-access and multi-operator systems. Simulation experiments, by using Network Simulator 2 (NS2) and real real-video sequences, were carried out in multimedia-aware IEEE 802.11e and IEEE $802.16 \mathrm{e}$ systems to show the impact of the proposed solution on the user's perception when compared with $\mathrm{MIH}$.

The remainder of the paper is organized as follows. Section II describes some related works. The QoEHand is explained in Section III. Section IV discusses the simulation experiments and results. Concluding remarks are given in Section V.

\section{RELATED WORK}

Challenges and requirements to create an IEEE 802.21 Media Independence Service Layer to optimize the usage of resources in heterogeneous wireless networks are discussed in [5]. The proposal uses a modular approach to coordinate mobility and video quality estimator components to provide seamless mobility. Our proposal uses the same modular (i.e., quality estimator, mapping and a set of adaptation components) and self-organized approach, but also adds and analyses the 
benefits of a QoE-aware MIH system on the user's perception A mobility server for seamless vertical handover in IEEE 802.21 MIH networks is proposed in [6]. Information about the wireless channel conditions is measured and used to provide seamless handover. However, the proposed solution does not take account of the existence of networks with different classes, which are expected in wireless systems. Moreover, it does not allow QoE assurance for users of multimedia content. A QoS architecture to provide a level of quality assurance for applications in heterogeneous environments is discussed in [7], while in [13] a systematic performance evaluation approach just focused on QoS parameters over heterogeneous wireless networks is presented. The proposal in [7] implements a schedule-based approach that draws on information about delay, loss and current network resources, and adjusts the scheduler to improve the video quality of delivery. However, this work does not provide seamless handover or follow its procedures in accordance with the user's experience/QoE (only QoS parameters are used, as in [13]). A MIH-based mobility scheme for IEEE 802.11 and IEEE $802.16 \mathrm{e}$ environments is discussed in [8]. The results showed that a wireless device can start its handover operation before the old link has been disconnected, and thus there is a reduction in packet losses and the latency. Another solution also uses a make-before-break scheme to support seamless mobility, but by using a Session Initiation Protocol (SIP)-based mobility management [9]. Our proposal follows the same make-before-break approach to provide seamless handover, but we also introduce the QoE video quality estimator, mapping and adaptation support, as required for heterogeneous networks. Few works have studied the benefits of an integrated QoE-aware wireless networking architecture with seamless mobility and heterogeneous support. This is undertaken in the next section where our proposal seeks to overcome the limitations of current proposals by allowing mobile users to be $\mathrm{ABC}$ with QoE support in multi-operator wireless environments.

\section{QOEHAND PROPOSAL}

The main objective of the QoEHand is to allow QoE-aware seamless mobility and optimization support for multimedia applications in heterogeneous networks. In this context, QoEHand agents are implemented, together with both Base Stations (BSs)/Access Points (APs) and wireless nodes, by following the recommendations of the MIH proposal. QoEHand extends MIH/IEEE 802.21 through the QoE-aware, mapping, video quality estimator and adaptation components. Well-defined interfaces and messages are used to allow a tight communication between IEEE 802.21 and QoEHand elements, such as improving the QoS schedulers with a QoE-aware dropping algorithm. Thus, wireless devices can be always best connected in heterogeneous and multi-operator networks and with QoE assurance. Its modular design allows the inclusion (or change) of policies, technologies and emerging services.

\section{III.A QOE VIDEO QUALITY ESTIMATOR}

A non-intrusive parametric video quality estimator is implemented by QoEHand agents to assess the quality level of video flows in IEEE 802.21 system (more details in [10]). A cluster-based Multiple Artificial Neural Network (MANN) mapping model is implemented to map video characteristics and network impairments (multi-operator heterogeneous systems) in MOS scores as a means of providing results that corresponded as closely as possible to human observer. The video quality estimator uses a set of feed-forward backpropagation networks that are supplied with subjective MOS scores. Thus, these parameters enable QoEHand to measure the quality level of videos even when they have different encoding patterns, genres, content types and packet loss rate. The results of the video quality estimator are used for mapping and adaptation handover decision. The video quality estimator uses objective parameters from the video encoder and wireless network conditions, as well as information about the perception of humans collected from the MOS experiments. The MOS is the most widely used metric and is recommended by the ITU BT. 500-11. The MOS is obtained by asking people to grade the quality of video on a five-point scale (Excellent, Good, Fair, Poor and Bad). The video quality estimator takes into account the current network conditions (different network impairments in the service classes) and different video parameters (percentage of losses in I, P and B frames, total number of losses, GoP length, and motion and complexity levels) that directly affect the quality of the video in terms of MOS prediction. As a result, this procedure gives a video quality score that corresponds as closely as possible to human perception in real-time. Since our proposal has been tested and validated, it is a dynamic and content-aware quality predictor that is able to estimate the video quality of several types of video content features in realistic multi-operator converged network conditions, without any interaction with real viewers. Figure 1 shows an overview of the video quality estimator, where original videos are encoded, transmitted in wireless networking systems (which have experienced network impairments) and the quality level of received videos are subjectively evaluated by real observers. For more details, please see [10].

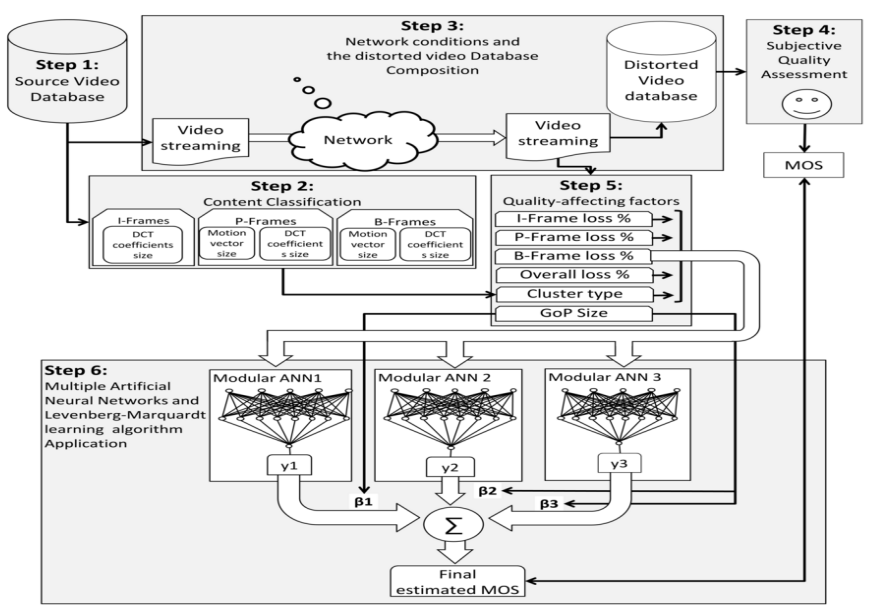

Fig 1. QoE video quality estimator.

This paper validated the video quality estimator in an IEEE 802.11e and IEEE 802.16e system, as explained in Section IV. After the observers had evaluated each video in service classes with different congestion levels, the training process was conducted by the training video database to obtain the mapping between the selected input (video/network) parameters and the 
MOS. The validation task was carried out with cross-validation techniques to reduce the generalization error.

If the predicted MOS indicates a low level of quality for the video flow, the QoEHand will search for a new (more suitable) class to map or adapt the multimedia content. The QoEHand considers the service classes available in the current and target networks so that it can offer continuous and seamless services and a satisfactory multimedia content delivery in heterogeneous networks, as will be expected in future systems.

\section{III.B QOE MAPPING MECHANISM}

The proposed QoE mapping mechanism maps application requirements and user's perception into available wireless service classes (IEEE 802.11e or IEEE 801.16 QoS models). The mapping process is carried out by drawing on information about the available service classes within or between networks (in multiple paths when possible), application QoS/QoE requirements, the video quality estimator score and mapping policies. The last of these decides which, what and when mapping methods must be used to carry out a request. After the mapping decision, the QoS scheduler is triggered to map the packets in the selected service class. The mapping policies define two main mapping methods to select the best class for an emerging multimedia application (its flows/components), called Full and Partial-Matching. A full-matching mapping is achieved when the quality level score of an application in a class is better than the minimal level. If there is more than one class result in the same quality level score, the policy scheme only considers the service class which has more available resources in terms of bandwidth. If the most suitable wireless service class is unable to assure a full matching (due to congestions or the existence of service classes with different configurations in terms of loss, delay and jitter support), the adaptation scheme is triggered to seek a potential adaptation for the applications that match the current network conditions. This adaptation can be carried out by intra-application adjustments or by requesting the re-mapping process with the aid of partial matching mapping rules. Depending on the business strategies, the nature of the multimedia content and the video quality level score, a set of dynamic partial matching mapping approaches can be applied as follows: (i) Downgrade class mapping: In this approach, a less important class is chosen to accommodate the application that assures a good/acceptable level of quality (video quality estimator score $>=$ minimal video quality level requirement); (ii) Scalable coding mapping: This approach takes into account the importance of each scalable flow (scalable video coding) of an emerging multimedia application during the mapping process. It maps high priority application flows into the best class and lower priority flows into a less significant class; (iii) Hierarchical component mapping: This approach selects service classes according to the order of priority of different multimedia components. Video communication is much more sensitive to packet loss than audio communication, because the human eye can often detect small glitches in a video stream caused by relatively minor packet loss, to the extent that enjoyment and/or understanding are more severely affected. For example, since voice has a higher priority than visual content in an application, the packets of audio flows are mapped to the best class and the packets of video flows to a lower priority class.

\section{III.C QOE ADAPTATION MECHANISM}

As mentioned earlier, one problem arising from multioperator wireless systems is the fact that each network provider can support different QoS models (e.g., IEEE 802.11e or IEEE 801.16) and can offer the same class of service with different definitions. For this reason, when the mapping process is not optimal (perfect match), the QoE Adaptation adjusts (e.g., downgrades) the quality level of the emerging applications if the network resources in a service class are unavailable (e.g., in congestion periods). The downgrade adaptation process is reversible when there are available resources in the previous service class again. In this case, the QoEHand can trigger MIH to handover the wireless client to the old network and maps all the flows into the previous service class. Since the success of our seamless proposal depends on adopting a make-beforebreak approach, the resources that are allocated and not used in previous or candidate service classes, are released by soft-state operations, for instance after a handover. A set of network adaptation profiles can be obtained by the adaptation mechanism to control the quality level of new or current applications. This is achieved as follows: 2D/3D Frame dropping adaptation (i): This approach drops packets in accordance with the visual importance of each frame encoded with common hierarchical 2D and 3D MPEG/H.264 codecs. I frames are marked with low dropping priority and B frames with high priority. Due to the intra-frame dependency on hierarchical codecs, when a P-frame is discarded, all of the subsequent $\mathrm{P}$ frames and $\mathrm{B}$ frames within the same GoP must also be discarded. When an I frame is discarded, all the other frames within the same GoP are dropped. Scalable video code adaptation (ii): This approach adjusts the quality level of applications by dropping or adding low important flows of scalable multimedia applications. Hierarchical component adaptation (iii): Media flows within an application should be marked with different priorities. Audio packets are marked with low priority and video packets with high dropping priority if voice content is more critical for the success/quality of the multimedia application. Region of Interest (ROI) - regions in the video sequence that are of most interest to the viewer (iv): This approach marks in-ROI (e.g., face) packets with low priority and out-ROI packets with a high dropping priority.

\section{III.D QOEHAND: MIH INTEGRATED ARCHITECTURE}

After introducing the functionalities of the mapping, adaptation, and video quality estimator, QoEHand will be described and integrated with an IEEE 802.21 system. In this system, each BS or AP (both with $\mathrm{MIH}$ ) gives information before the connection (both IEEE 802.11e and IEEE 802.16), about which service class (including the current channel conditions in terms of loss, delay and bandwidth) are available to connect the applications of the wireless devices. This information is used as input for the video quality estimator to define a MOS for the video services in the available classes. After selecting a class (e.g., Access Category 1 in IEEE 802.11e or Unsolicited Grant Service (UGS) in IEEE 802.16e), the BS/AP establishes a connection linked to the user in a QoS class with enough resources to ensure a quality level that is 
suitable for the application. For cost reasons (it can be easily configured by the administrator), QoEHand will try to map the video flows in IEEE 802.11e networks before to handover to IEEE 802.16e systems in case of failure (not a perfect match).

The MIH establishes communication between the lower and upper layers, on the basis of a set of IEEE 802.21 primitives defined as SAPs (Service Access Points). There are three SAPs as follows: MIH_SAP, MIH_NET_SAP and MIH LINK SAP. The MIH LINK SAP is responsible for giving information about the service class parameters of the MAC layer technologies to the upper layers. QoEHand will use this information for quality estimation, mapping and adaptation procedures. The mapping can increase the user's perception and guarantee the quality level support in both the IEEE 802.11e and IEEE 802.16e systems. Based on the QoEHand output (e.g., video quality score), the MIH module in the mobile client is triggered to choose one (or a new) network to be connected, where all video flows are be mapped or adapted to the best service class.

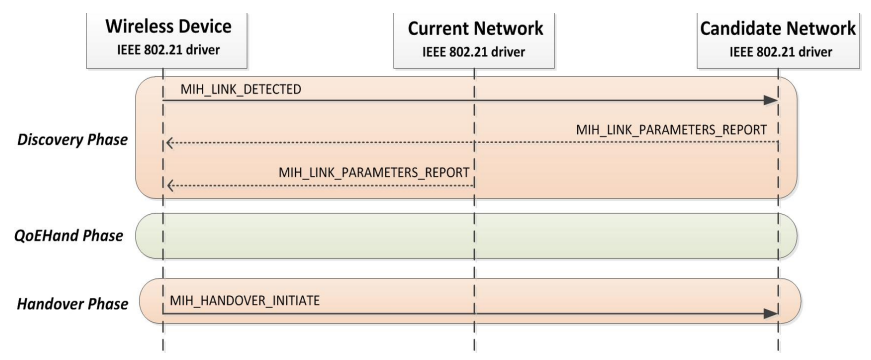

Fig 2. Signalling messages and operations in a handover .

When a wireless node detects a Target/Candidate/Foreigner Network, the MIH module sends a MIH_LINK_DETECTED message to inform the Target Network that there is a new client in a coverage area as presented in Figure 2 (discovery phase). The Target Network sends a MIH_LINK_PARAMETERS_REPORT to wireless device, where the available classes and their conditions are informed. After that, the QoEHand (QoEHand phase) in the Current Network measures and compares the level of video quality in the current and foreign classes (by using the video quality estimator) and triggers the mapping mechanism to select the best service class for the multimedia applications. If the quality level score in the target network is higher, QoEHand informs the mobile node (by using internal interfaces) about a handover decision. The seamless handover is initiated by using a MIH HANDOVER INITIATE. The handover can also be triggered in congestion periods when the video quality estimator detects a new class in a target network that can assure a better level of quality for current video flows. If a full match is not possible, the adaptation mechanism is requested to adjust the application quality level to the current channel conditions.

\section{PERformance Evaluation AND RESUlts}

Simulation experiments were carried out by using the Network Simulator 2 (NS2) and Evalvid (to control the video distribution). The objective was to analyse the benefits of QoEHand and its impact on IEEE 802.11e and IEEE 802.16e networks, compared with a system without QoEHand (without video prediction, mapping and adaptation - and only with MIH functionalities), by measuring QoE subjective (MOS) and objective (Video Quality Metric - VQM) metrics. The VQM values vary from 0 to 5 , where 0 is the best possible score. The QoEHand video quality estimator was formed with the aid of MATLAB. The RTP payload header includes a field that indicates the current frame type, i.e., I, P, or B frames.

Four profiles were configured in the system to determine the benefits of QoEHand with different scenarios and experiments: (i) Pure_MIH (without QoEHand); (ii) QoEHand_Full, when a full mapping match is achieved during the handover and there are available resources in the service class of the foreign network; (iii) QoEHand_Part profile, which re-maps all the packets of a video sequence into a less important class in the target network, because the most suitable wireless service class cannot assure a full-matching (e.g., due to congestions); (iv) QoEHand_Drop which controls the video quality level by dropping video packets in descending order of importance, from the standpoint of the user's perception. The ITU-T MOS recommendation was used for a subjective evaluation with 55 observers. They had normal vision and their ages ranged from 18 to 45 (undergraduate students, postgraduate students, and university staff). The SingleStimulus (SS) method was used in the experiments, because it is suitable for large-scale tests, where a processed video sequence is displayed by itself, without being paired with its unprocessed reference version. The test platform used was a Desktop PC with Intel Core i5, 4GB RAM and a 21" LCD monitor. A software tool was implemented to display the video sequences and collect the user's scores. 10 different wellknown Internet video sequences were selected for the experiments (Akiyo, Container, Coastguard, Highway, Football, Hall, Mobile, Grandma, News, Silent) with different levels of complexity and motion [11]. The video sequences were encoded in MPEG4 format and the duration varied from $10 \mathrm{~s}$ to $30 \mathrm{~s}$. The GoP length was 18 , which is what can be expected for common Internet video streaming. To provide a large enough video database and increase the reliability of the system, each selected video was simulated 10 times by varying the congestion periods (from $0 \%$ to $50 \%$ in steps of 5 ) in a service class, resulting in a total of 100 (received) videos with a different packet loss rate. A multi-operator scenario is used to evaluate the QoEHand. QoEHand can work on both fixed and mobile systems, where handovers can be triggered due to the movement of a mobile device to a new AP/BS or due to congestions in a service class. In order to simplify the experiments, we assume that the wireless nodes are fixed, implement both IEEE 802.11e and IEEE 802.16e interfaces and handover to a new network or service class due to congestions. Two service classes were configured in each network (IEEE 802.11e - AC V0 and AC V1 / IEEE 802.16e - Real-time Polling Service (rtPS) and Non-real-time Polling Service (nrPS). The service class, in which the user is receiving the video in the current network, will experience congestions of from $5 \%$ to $50 \%$ in steps of 5 by concurrent traffic. Hence, QoEHand will interact with $\mathrm{MIH}$ to adjust (handover, remapping or drop packets) the video quality level based on one of its 3 profiles (QoEHand_Full, QoEHand_Part and QoEHand_Drop). 10 simulations were carried out for each video, where, in five cases, the receiver experienced congestions in a service class of an IEEE 802.11e network. In 
the other 5 experiments, the receiver experienced congestions in a service class of an IEEE 802.16e network.

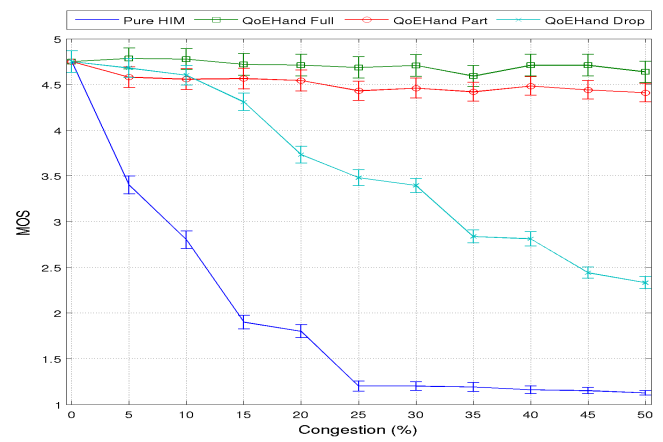

Fig 3. Congestion x MOS for all the profiles.

As illustrated in Figure 3, the MOS results show that QoEHand assures an excellent quality level for the videos during congestion periods when the QoEHand_Full and QoEHand_Part profiles are used. In the QoEHand_Part, the videos still have a good-to-excellent quality level even when re-mapped to a less important class (nrPS or AC V1) with a packet-loss rate of approximately 2\%. The QoEHand_Drop attempts to keep the application at an excellent quality level of up to $10 \%$ of congestion and at a good/regular quality level of up to $30 \%$ of congestion. However, when the Pure_MIH is used, the video quality level was considered poor by all the observers, if there was a minimum of $10 \%$ of congestion in a wireless service class. When an I frame is dropped, the error is spread through the rest of the GoP and the quality is bad/poor, because the MPEG decoder uses the I frame as a referencepoint for all the other frames within a GoP.

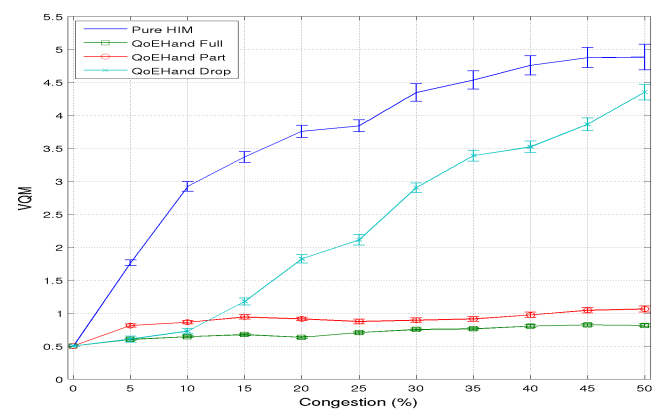

Fig 4. Congestion $\mathrm{x}$ VQM for all the profiles.

The VQM results for all the tests are shown in Figure 4 and demonstrate the benefits of QoEHand profiles in a QoE-aware converged wireless network (e.g., by analysing blurring, global noise, block distortion and the colour distortion of the videos). The QoEHand_Full profile kept the VQM values at around 0.75 throughout the experiments. Compared with the QoEHand_Full profile the QoEHand_Part, on average, reduced the video quality level by 0.3 for all the simulations. In congestion periods of a service class, the QoEHand Drop keeps the VQM values at less than 1 when the congestion rises to $10 \%$. Since the B and $\mathrm{P}$ frames are discarded first, the impact on the user's perception is kept to a minimum when the system is configured with QoEHand_Drop. If it exceeds a congestion rate of $5 \%$, the Pure $\mathrm{MIH}$ profile can no longer assure the minimal quality level for the videos.

\section{CONCLUSION AND FUTURE WORK}

Emerging multimedia wireless networks will allow users to be connected to the best QoE-aware wireless access network, where seamless mobility will be combined with respect for the user's preferences. This paper proposes a modular architecture called QoEHand to maximize the QoE of wireless clients in MIH IEEE 802.11 and 802.16 systems, by coordinating quality estimator, mapping and adaptation mechanisms. Simulation experiments were carried out to show the impact and benefits of QoEHand in an IEEE 802.11e and IEEE 802.16e multioperator system when compared with a MIH scheme. In future studies, QoEHand will be an analysis of a mobile dynamic scenario with dozens of mobile users competing for resources in IEEE 802.11e, 802.16e and LTE.

\section{ACKNOWLEDGEMENT}

This work was funded by: 1) National Council for Scientific and Technological Development (CNPq); 2) Fundação Amazônia Paraense de Amparo à Pesquisa (FAPESPA); 3) FCT UBIQUIMESH project (PTDC/EEATEL/105472/ 2008); 4) PLATINO project financed by MIUR; and 5) LINCE project of the FARO programme jointly funded by the Compagnia di San Paolo and by the Polo delle Scienze e delle Tecnologie of the University of Napoli, Federico II.

\section{REFERENCES}

[1] De La Oliva et all, "IEEE 802.21: Media independence beyond handover", Computer Standards \& Interfaces Vol. 33, Issue 6, pp. 556-564, Nov. 2011.

[2] Jingjing Zhang; Ansari, N.; "On Assuring End-to-End QoE in Next Generation Networks: Challenges and a possible Solution", Communications Magazine IEEE, vol 49, no 7, pp. 185-191, July 2011.

[3] Aguiar, E at all, "Real-Time QoE Prediction for Multimedia Applications in Wireless Mesh Network", $4^{\text {th }}$ IEEE Future Multimedia Network Workshop, Las Vegas, USA, Jan 2012.

[4] Matos, F. at all, " QoS adaptation in inter-domain services ", 12th IFIP/IEEE International Symposium on Integrated Network Management, IM 2011, Dublin, Ireland, May 2011.

[5] Jingjing, Z.; Ansari, N., "On assuring end-to-end QoE in next generation networks: challenges and a possible solution", IEEE Communications Magazine. Vol. 49, Issue: 7, 2011.

[6] Kim, Y. et all, "An enhanced information server for seamless vertical handover in IEEE 802.21 MIH networks", IEEE Computer Networks, vol 55, Issue 1, January 2011.

[7] Liang, Z.; Han-Chieh, C.; Vasilakos, A.V., (2011). "Joint ForensicsScheduling Strategy for Delay-Sensitive Multimedia Applications over Heterogeneous Networks", IEEE JSAC, Vol 29, Issue 7, August.

[8] Lim, Wan-Seon et all, "Implementation and performance study of IEEE 802.21 in integrated IEEE 802.11/802.16e networks", Computer Communications, Vol 32, Issue 1, January 2009.

[9] Yulong Shen et all, "MS2HM: A Multimedia Service Session Handoff Method between Heterogeneous Wireless Networks", IEEE INCoS, Fukuoka, Japan, Dec 2011.

[10] Aguiar, E at all,"Video quality estimator for wireless mesh networks ", IEEE/ACM 20th International Workshop on Quality of Service (IEEE/ACM IWQoS 2012), Coimbra, Portugal, June 2012.

[11] "Video trace library," Available in trace.eas.asu.edu, accessed in 2012.

[12] M. Bernaschi, F. Cacace, A. Pescapè, S. Za, "Analysis and Experimentation over Heterogeneous Wireless Networks", IEEE TRIDENTCOM 2005 pp. 182-191, Trento (Italy), February, 2005.

[13] A. Botta, D. Emma, A. Pescapè, G. Ventre, "Systematic Performance Modeling and Characterization of Heterogeneous IP Networks", Journal of Computer and System Sciences (Elsevier) - Vol. 72, Is. 7, Nov. 2006, pp 1134-1143. 